\title{
Development of Personality Disorders: Identity as a Key Process
}

\author{
Ilaria Maria Antonietta Benzi*, Fabio Madeddu
}

Department of Psychology, University of Milano-Bicocca, Piazza dell'Ateneo Nuovo 1, 20126, Milano, Italy

\section{Abstract}

Currently, researchers and clinicians are conducting a wide-ranging reflection on personality disorders (PDs): significant aspects on this topic are the need to delineate the core domains that define personality pathology, as well as the demand to formulate a clinically relevant description of PDs. Within a dimensional perspective, given the evidence of a strong relationship between identity pathology and PDs, identity emerges as a key process, considering both the subjective experience (integration vs. diffusion) and the social-cognitive perspectives (degrees of development of identity processes), in unravelling aspects of the self and interpersonal impairment in personality pathology.

\section{Publication History:}

Received: February 02, 2016

Accepted: March 07, 2017

Published: March 09, 2017

\section{Keywords:}

DSM-5 Alternative Model for PDs, Interpersonal impairment, Personality traits, Self impairment

\section{Introduction}

Personality disorders (PDs) are defined as patterns of maladaptive personality traits that have an onset during childhood or adolescence and then impact significantly on the individual's life. Westen \& Chang [1] emphasized some issues that continue to challenge our understanding, among them the need to delineate what the core domains are that define personality in adolescence; and what kind of development, normal and pathological, we can expect during this period of life. Although we currently lack a deep understanding of the steps that lead to the development of PDs, research highlights specific developmental models that underline major key processes involved in the development of personality pathology $[2,3]$.

Within this context, the definition of identity and the hypotheses on its formation (IF) emerged as important aspects to consider in order to deepen the understanding of PDs. In fact, evidence of a strong relationship between identity pathology and PDs was found: impairment in identity integration resulted predictive of PDs in adults [4] as well as identity disturbance in adolescents (lack of normative commitment and consistency, role absorption and incoherence) has proven to be related with different forms of personality pathology [5]. Also, research investigated the role of both identity and personality traits in the development of personality: the significance of personality traits for psychological development was emphasized [6] as well as a mutual interaction between identity and personality traits was demonstrated [7]. Besides, identity impairment seems to play a significant role in understanding higher-order constructs that are related to PDs, such as externalizing and internalizing [8]: behavioral and emotional difficulties in adolescence are related to internalizing aspects, such as depression and anxiety, and externalizing symptoms, such as aggression [9], whilst identity processes, like exploration and commitment, result negatively associated with internalizing features [10].

\section{Identity Domains}

The concept of identity has undergone different formulations over the years, starting from the first conceptualization proposed by Erikson that described the construct of identity as normal ego identity, which permits the development of an adaptive personality; identity crisis, which is typical of adolescence, challenging the adolescents to update self and interpersonal features according to the developmental shifts they are facing; and identity diffusion, a significant impairment in experiencing an integrated self and clear boundaries between self and others, that is linked to PDs [11].
Later revisions of the concept can be grouped into two main domains. On the one hand, psychoanalytic and psychodynamic theories refer to identity as a subjective experience emphasizing its continuity/discontinuity and its emotional aspects. On the other, social-cognitive perspectives investigate identity related to its interpersonal reflections focusing on coherence, cognitive access and social indicators [12].

According to the former domain, O. Kernberg [13] formulated an approach that considers the subjective aspects of identity, suggesting a dimensional model of personality that integrates identity, psychic defenses, and reality testing in order to delineate different levels of functioning (neurotic, borderline and psychotic). Identity diffusion, which corresponds to non-integrated, positive and negative, internalized aspects of the self, is considered one of the key process of personality pathology [13]. As pointed out by M.P. Kernberg et al. [14] the clinical manifestations of identity pathology in adolescence have an influence both on self-functioning, affecting their ability to express themselves, to develop and maintain life goals and interests, and interpersonal functioning, implying difficulties in establishing and maintaining relationships, in empathizing and in the ability to understand other people perspectives.

A development of the social-cognitive viewpoint is Marcia's [15] which acknowledged two key processes of IF: exploration, demonstrating how adolescents are able to experiment themselves among different levels of commitment within the domains of identity; and commitment, which explains how youths make choices regarding their own identity and are actually committed to them. Depending on the level of exploration and commitment, Marcia delineated four identity statuses: identity diffusion (lack of commitment related to a developmental task), foreclosure (not enough exploration), in moratorium (exploration but no significant commitment) and identity achievement (exploration is completed and commitment is fully achievable).

"Corresponding Author: Dr. Ilaria Maria Antonietta Benzi, Department of Psychology, University of Milano-Bicocca, Piazza dell'Ateneo Nuovo 1, 20126, Milano, Italy; E-mail: ilaria.benzi@unimib.it

Citation: Benzi IMA, Madeddu F (2017) Development of Personality Disorders Identity as a Key Process. Int J Psychol Behav Anal 3: 124. doi: https://doi. org/10.15344/2455-3867/2017/124

Copyright: (C) 2017 Benzi et al. This is an open-access article distributed under the terms of the Creative Commons Attribution License, which permits unrestricted use, distribution, and reproduction in any medium, provided the original author and source are credited. 
Both the approach that focuses more on identity of the self and the social-cognitive perspective, highlight a dimensional conceptualization of the construct: on the one hand, in terms of level of integration, on the other, related to a certain degree of development of different identity processes [16].

\section{Toward a model for personality disorders: the role of identity}

Currently, the scientific community is involved in a wide-ranging reflection on PDs: in particular, research tries to frame core aspects and to formulate a clinically relevant description of PDs [17]. In this regard, during the last decade clinicians and researchers have been involved in a debate on psychopathological personality structures with the aim of overcoming the limitations of the diagnostic system proposed by the DSM-IV-TR [18] (e.g. co-occurrence among PDs, clinical heterogeneity, temporal instability) and accounting for the evidence that severity of the impairment is the most significant predictor of concurrent and forthcoming dysfunctions [19].

An answer to these limitations is coming from both the major diagnostic systems, DSM and ICD, with the formulation of a dimensional approach, considering the severity of the personality impairment and recommending a revision of the central aspects of the disorder [20]: DSM-5, while maintaining the previous classification for PDs, suggests in Section III (Emerging Measures and Models) an hybrid model for PDs; instead, the proposed ICD-11 classification eliminates the previous specific PDs and promotes a dimensional assessment of the personality disorder itself [21].

An answer to these limitations is coming from both the major diagnostic systems, DSM and ICD, with the formulation of a dimensional approach, considering the severity of the personality impairment and recommending a revision of the central aspects of the disorder [20]: DSM-5, while maintaining the previous classification for PDs, suggests in Section III (Emerging Measures and Models) an hybrid model for PDs; instead, the proposed ICD-11 classification eliminates the previous specific PDs and promotes a dimensional assessment of the personality disorder itself [21].

While both the Alternative Model of DSM- 5 and the proposal for the ICD-11 consider a combination of pathological traits in the evaluation of personality pathology, a distinct feature of the DSM5 hybrid model is the core aspect of personality pathology: in fact, DSM-5proposesto assess impairment in self-functioning (deficits in identity and self-direction) and in interpersonal functioning (deficits in empathy and intimacy) as central features of PDs. Such impairments are measured along a scale ranging from little or no-impairment up to extreme impairment. Besides the evaluation of the Level of Personality functioning, DSM-5 introduces twenty-five specific trait facets that are organized into five main domains (negative affectivity, detachment, antagonism disinhibition vs. compulsivity and psychoticism): a growing amount of research follows this approach and, although adolescence is a stage of development marked by intense fluctuations involving behavioral, cognitive and affective aspects, demonstrates how personality traits are characterized by an overall stability during the transition from childhood to adulthood and might be clinically more relevant than sets of symptoms in understanding PDs [22]. Such a dimensional approach allows the pathology of personality itself to be considered as an extreme manifestation of impairment that lies along a continuum that includes different levels of severity. Thus, the assessment of personality functioning can be linked to the key processes that are maladaptive both on an intra-psychic and interpersonal level rather than just to symptomatic manifestation: indeed, the hybrid model proposes five specific PDs (antisocial/ psychopathic, avoidant, borderline, obsessive-compulsive, and schizotypal), each one of those is characterized by an impairment in personality functioning and is described with a trait list that specify its fundamental pathological personality traits [19]. Furthermore, this approach has shown an increase in the stability between the patterns of personality found in adolescence through adulthood [23].

This kind of model helps to highlight the crucial role of identity in understanding PDs: in fact, as a feature of the impairment of the self, identity is the subject's ability to have a unique experience of the self and also to have clear boundaries between self and others. This aspect seems to be critical in influencing other parts of personality pathology: an integrated identity influences the subject both in pursuing goals in life and in self reflecting productively (self-direction) and in understanding other people perspectives (empathy) and forming close relationships (intimacy) [24].

\section{Conclusions}

Due to the assumption of a dimensional perspective, research and clinical experience seem to emphasize the complexity of the role identity plays in understanding PDs and open up challenging lines of research.

Firstly, different dimensions of the identity construct, such as the subjective experience (integration vs. diffusion) and the socialcognitive perspectives (degrees of development of identity processes), seem to properly unravel aspects of the self and interpersonal impairment in personality pathology. In addition, this consideration appears to underline the clinical utility of considering both dimensions of identity together trying to understand whether the subject has an integrated image of himself and how this might affect the level of exploration and commitment [25].

In the light of this, another promising area of study might be the exploration of the mutual relationship between identity and personality traits, given that research has shown how configurations of maladaptive traits are linked to self and interpersonal difficulties [26].

\section{Competing Interests}

The authors declare that they have no competing interests.

\section{References}

1. Westen D, Chang C (2000) Personality pathology in adolescence: A review. Adolescent Psychiatry 25: 61-100.

2. De Fruyt F, De Clercq B (2014) Antecedents of personality disorder in childhood and adolescence: toward an integrative developmental model. Annul Rev of Clinical Psychology 10: 449-476.

3. Ensink K, Biberdzic M, Normandin L, Clarkin J (2015) A Developmental Psychopathology and Neurobiological Model of Borderline Personality Disorder in Adolescence. Journal of Infant, Child, and Adolescent Psychotherapy 14: 46-69.

4. Skodol AE, Bender DS, Oldham JM, Clark LA, Morey LC, et al. (2011b) Proposed changes in personality and personality disorder assessment and diagnosis for DSM-5 Part II: Clinical application. Personality Disorders: Theory, Research, and Treatment 2: 23.

5. Westen D, Betan E, DeFife JA (2011) Identity disturbance in adolescence: Associations with borderline personality disorder. Development and psychopathology 23: 305-313 
Citation: Benzi IMA, Madeddu F (2017) Development of Personality Disorders: Identity as a Key Process. Int J Psychol Behav Anal 3: 124. doi: https://doi. org/10.15344/2455-3867/2017/124

Page 3 of 3

6. Klimstra TA (2012) The dynamics of personality and identity in adolescence. European Journal of Developmental Psychology 9: 472-484.

7. Luyckx K, Soenens B, Goossens L (2006) The personality identity interplay in emerging adult women: convergent findings from complementary analyses. European Journal of Personality 20: 195-215.

8. Luyten P, Blatt SJ (2011) Integrating theory-driven and empirically-derived models of personality development and psychopathology: a proposal for DSM V. Clin Psychol Rev 31: 52-68.

9. Besser A, Blatt SJ (2007) Identity consolidation and internalizing and externalizing problem behaviors in early adolescence. Psychoanalytic psychology 24: 126-129.

10. Jung E, Pick O, Schlüter-Müller S, Schmeck K, Goth K (2013) Identity development in adolescents with mental problems. Child Adolesc Psychiatry Ment Health 7: 26 .

11. Erikson EH (1959) The theory of infantile sexuality. In Childhood and Society (pp. 42-92). New York: W.W. Norton \& Co.

12. Foelsch PA (2014) Adolescent Identity Treatment: An Integrative Approach for Personality Pathology. Springer.

13. Kernberg OF (1998) The diagnosis of narcissistic and antisocial pathology in adolescence. Adolescent Psychiatry 22: 169.

14. Kernberg PF, Weiner AS, Bardenstein KK (2008) Personality disorders in children and adolescents. Basic Books.

15. Kroger J, Marcia JE (2011) The identity statuses: Origins, meanings, and interpretations. In Handbook of identity theory and research (pp. 31-53) Springer New York.

16. Meeus W (2011) The study of adolescent identity formation 2000-2010: A review of longitudinal research. Journal of research on adolescence 21: 75-94

17. Clarkin JF, Meehan KB, Lenzenweger MF (2015) Emerging approaches to the conceptualization and treatment of personality disorder. Canadian Psychology/Psychologie canadienne 56: 155-167.

18. Skodol AE (2012) Diagnosis and DSM-5: Work in progress. The oxford handbook of personality disorders. (pp. 35-57) Oxford University Press, New York, NY

19. Skodol AE, Clark LA, Bender DS, Krueger RF, Morey LC, et al. (2011) Proposed changes in personality and personality disorder assessment and diagnosis for DSM-5 Part I: Description and rationale. Persona Disord 2 : 4-22.

20. American Psychiatric Association (2013) Diagnostic and statistical manual of mental disorders (5th edn.). Washington, DC: Author.?

21. Tyrer P, Reed GM, Crawford MJ3 (2015) Classification, assessment, prevalence, and effect of personality disorder. Lancet 385: 717-726.

22. Luyckx K, Teppers E, Klimstra TA, Rassart J (2014) Identity processes and personality traits and types in adolescence: directionality of effects and developmental trajectories. Dev Psychol 50: 2144-2153.

23. Stepp SD (2012) Development of borderline personality disorder in adolescence and young adulthood: Introduction to the special section. J Abnorm Child Psychol 40: 1-5.

24. Schmeck K, Schlüter-Müller S, Foelsch PA, Doering S (2013) The role of identity in the DSM- 5 classification of personality disorders. Child Adolesc Psychiatry Ment Health 7: 27 .

25. Wright AG, Pincus AL, Hopwood CJ, Thomas KM, Markon KE, et al. (2012) An interpersonl analysis of pathological personality traits in DSM-5 Assessment 19: 263-275. 